\title{
Article
}

\section{Nursing judgement and decision-making using the Sedation Withdrawal Score (SWS) in children}

Craske, Jennie, Carter, Bernie, Jarman, Ian H. and Tume, Lyvonne Nicole

Available at http://clok.uclan.ac.uk/17861/

Craske, Jennie, Carter, Bernie ORCID: 0000-0001-5226-9878, Jarman, Ian H. and Tume, Lyvonne Nicole ORCID: 0000-0002-2547-8209 (2017) Nursing judgement and decision-making using the Sedation Withdrawal Score (SWS) in children. Journal of Advanced Nursing, 73 (10). pp. 2327-2338. ISSN 03092402

It is advisable to refer to the publisher's version if you intend to cite from the work. http://dx.doi.org/10.1111/jan.13305

For more information about UCLan's research in this area go to

http://www.uclan.ac.uk/researchgroups/ and search for <name of research Group>.

For information about Research generally at UCLan please go to http://www.uclan.ac.uk/research/

All outputs in CLoK are protected by Intellectual Property Rights law, including Copyright law. Copyright, IPR and Moral Rights for the works on this site are retained by the individual authors and/or other copyright owners. Terms and conditions for use of this material are defined in the policies page. 
NURSING JUDGEMENT AND DECISION-MAKING USING THE SEDATION WITHDRAWAL SCORE IN CHILDREN 


\section{ABSTRACT}

\section{Aims}

The aim of the study was to evaluate registered children's nurses' approaches to the assessment and management of withdrawal syndrome in children.

\section{Background}

Assessment of withdrawal syndrome is undertaken following critical illness when the child's condition may be unstable with competing differential diagnoses. Assessment tools aim to standardise and improve recognition of withdrawal syndrome. Making the right decisions in complex clinical situations requires a degree of mental effort and it is not known how nurses make decisions when undertaking withdrawal assessments.

\section{Design}

Cognitive interviews with clinical vignettes.

\section{Methods}

Interviews were undertaken with 12 nurses to explore the cognitive processes they used when assessing children using the Sedation Withdrawal Score (SWS) tool. Interviews took place in Autumn 2013.

\section{Findings}

Each stage of decision-making - noticing, interpreting and responding - presented cognitive challenges for nurses. When defining withdrawal behaviours nurses tended to blur the boundaries between Sedation Withdrawal Score signs. Challenges in interpreting behaviours arose from not knowing if the patient's behaviour was a result of withdrawal or other comorbidities. Nurses gave a range of diagnoses when interpreting the vignettes, despite being provided with identical information. Treatment responses corresponded to definite withdrawal diagnoses, but varied when nurses were unsure of the diagnosis. 


\section{Conclusion}

Cognitive interviews with vignettes provided insight into nurses' judgement and decisionmaking. The SWS does not standardise the assessment of withdrawal due to the complexity of the context where assessments take place and the difficulties of determining the cause of equivocal behaviours in children recovering from critical illness.

\section{KEY WORDS}

cognitive interviewing, critical care, paediatric, nursing assessment, cognitive errors 


\section{SUMMARY STATEMENT}

\section{Why is this research or review needed?}

- There is little research that considers how children's nurses make decisions in complex clinical situations with regards to assessment of withdrawal syndrome.

- Assessment of withdrawal syndrome is challenging due to competing differential diagnoses and inaccurate assessment may lead to unnecessary changes to a child's treatment.

\section{What are the key findings?}

- Making the right decisions in complex clinical situations is not simple and the Sedation Withdrawal Score tool did not support the depth of thinking necessary to successfully negotiate the confounding factors.

- Once a validated tool is adopted into clinical practice the challenge is that individual practitioners think differently and at different levels to each other and this is rarely accounted for in the development of scoring tools to support clinical decisions.

- Not every practitioner will approach the complexity of the task with the same level of critical thinking.

\section{How should the findings be used to influence policy/practice/research/education?}

- Complex clinical situations cannot be made simpler but users of tools can be supported to improve their critical thinking; incorporating metacognition into education is one approach.

- Multiple scoring tools are used in healthcare, yet the way these are actually used is rarely studied. If tools are to achieve their intended outcomes then more research should be undertaken to explore the way they are used. 


\section{INTRODUCTION}

Children admitted to the paediatric intensive care unit (PICU) require adequate sedation and analgesia for the duration of their critical illness (Jenkins 2002). Many of these drugs cause physical dependence, which means that once the child is recovering the drugs should to be tapered, rather than being stopped abruptly to prevent withdrawal syndrome (Cunliffe et al. 2004, Easley \& Nichols 2008). Although each drug has a distinctive withdrawal syndrome, many of the signs of withdrawal are the same across drug groups. Individually however, these equivocal signs are ambiguous in the critically ill child, possibly indicating pain, delirium, the underlying condition, deterioration or withdrawal (Harris et al. 2016)

\section{BACKGROUND}

The assessment of withdrawal syndrome in children is complex. Structured and repeatable assessments are recommended to assist detection, but it is often unclear how these are applied by nurses. Three published tools have been developed to monitor withdrawal in children; the Sedation Withdrawal Score (SWS) (Cunliffe et al. 2004), the Withdrawal Assessment Tool (WAT-1) (Franck et al. 2008) and the Sophia Observation Score (SOS) (Ista et al. 2009). Each is a checklist of non-specific signs that, in combination, appear to support a diagnosis of withdrawal (Table 1). The SWS is the withdrawal assessment tool and treatment protocol used in our hospital since 2004. SWS has proven clinically useful in identifying withdrawal signs in ICU and ward-based patients, but has not been validated (Macqueen \& Bruce 2012). Both WAT-1 (Franck et al. 2008, 2012) and SOS (Ista et al. 2009, 2013) have been validated but the studies excluded patients whose existing behaviour might confound the withdrawal assessment and clinical utility is further limited by the lack of linked treatment protocols. 
The assessment is complex due to the multiple drug and patient factors to be considered. Drug factors include the likelihood of physical dependence, which varies depending on drug dose and duration of therapy (Amigoni et al. 2014, da Silva et al. 2016) but also appears highly individualised (Best et al. 2016) and may be further complicated by concurrent tapering of more than one sedative or analgesic drug. Patient factors include the highly individualised effects of withdrawal on the child's recovery (Franck et al. 2008) and the confounding effect of the patient's primary medical condition on withdrawal intensity (Franck et al. 2008). Assumptions are made that the nurse will modify the assessment to ensure the underlying condition or any external factors do not skew the withdrawal score (Franck \& Vilardi 1995, Ista et al. 2013, Harris et al. 2016). Complex tasks like this demand a degree of cognitive effort and focussed attention on the part of the nurse, to make correct judgments and decisions. Given that the judgement of the bedside nurse has been a 'silver standard' in tool validation studies (Franck et al. 2008, Ista et al. 2009), it is important to understand how nurses think when undertaking withdrawal assessments and making treatment choices (Easley \& Nichols 2008).

Two key theoretical approaches to clinical decision-making are reasoning and intuition (Banning 2008). Different academic disciplines have investigated the analytical, rational approach of reasoning and similar models have emerged to describe the key stages of the process (Table 2). Remaining open minded throughout these stages is vital, as relevant cues can be subtle and may be overlooked, particularly if the situation is changing quickly or there is too much to take in simultaneously (Gaba et al. 1995). Knowing how to filter tenuous cues and focus on relevant ones is a feature of expert nursing practice (Harbison 2006).

Intuition is defined as 'a way of knowing something immediately as a whole that improves with experience' (Rew \& Barrow 2007, p. E25). This enables the clinical expert to process and identify key diagnostic components subconsciously (Lyneham et al. 2008). In high- 
pressure circumstances, the rational approach is somewhat idealistic as clinical decisions are often required despite incomplete knowledge of the situation (Graber et al. 2002). Under these conditions, experienced clinicians rely on intuition to 'think fast' (Kahneman 2011, p. 13), using pattern recognition(Berner \& Graber 2008, Gobet \& Chassy 2008) and heuristics ( Elstein 1999, Cranley et al. 2009). However, intuitive processing can be flawed (Graber et al. 2005), especially in an unpredictable environment (Kahneman \& Klein 2009) such as critical care.

No published papers were identified that considered how nurses make decisions about the assessment and management of withdrawal in children. This study sought to fill this knowledge deficit by attending to the three stages of decision making; noticing, interpreting and response.

\section{AIM}

The aim of the study was to explore registered children's nurses' decision-making during the assessment and management of withdrawal in children by examining:

1. Noticing: the nurses' recognition and understanding of four clinical signs from the SWS tool.

2. Interpreting: the meaning of an SWS score, in terms of a diagnosis of withdrawal, presented in two clinical vignettes;

3. Response: the treatment choices made in response to the withdrawal diagnosis

\section{DESIGN}

Cognitive interviews were undertaken using clinical vignettes to explore the study aims. 
Cognitive interviews are a recognised approach to explore cognitive processing in relation to decision-making (Willis 2005, Ross et al. 2012). The fundamental features of cognitive interviews are think aloud and verbal probing: techniques which permit the researcher to listen in to the complex and usually hidden evolution of (clinical) reasoning without interfering with the cognitive processes being uncovered (Fonteyn et al. 1993). Verbal probing delivered in a neutral manner enables the interviewer to drill down on the issues under investigation, so clinical expertise in the subject area is necessary to recognise when a response needs further probing (Sofaer 2002). All other interaction between researcher and participant is minimised to reduce biasing the participants' responses (Sofaer 2002).

The cognitive interview approach has been employed in other studies investigating nurse decision-making (Cioffi 1998, Simmons et al. 2003, Twycross \& Powls 2006, Hoffman et al. 2009); these are now presented in brief. Cioffi (1998) investigated the effects of experience and uncertainty on triage assessments made by emergency nurses and Simmons et al. (2003) described cognitive processes used by experienced nurses during their patient assessments in elderly care. Work by Twycross \& Powls (2006) explored how children's nurses made clinical decisions and Hoffman and colleagues (2009) compared clinical cues collected by novice and expert nurses in intensive care. The cognitive interview approach has also been applied to the psychometric testing of self-report clinical assessment tools, to check that terminology is understood and interpreted consistently by patients ( Sofaer 2002, DiBenedetti et al. 2013), so was well suited to the aims of this study.

An experimental setting, using vignettes, was chosen over a naturalistic setting to control the clinical data provided to participants and allow comparison between them (Willis 2005, Berner \& Graber 2008). By standardising the data, the only variable lay in the nurses' decision making processes (Cook \& Rumrill 2005), enabling focus on the abstruse stages of 'noticing' and 'interpreting' rather than simply the outcome or response (Veloski et al. 2005). 
Clinical vignettes were developed by JC (an experienced pain/sedation nurse specialist) to illustrate a typical, complex clinical situation featuring a patient with severe neurological disability (Figure 1). The vignettes were based on a real case from clinical practice to enhance believability (Endacott et al. 2010). Face and content validity were assessed by four senior clinical nurses (members of the Pain and Sedation Service and an Advanced Nurse Practitioner in critical care) experienced in withdrawal assessment (Brattebo 2009).

Typical levels of cognitive stimulation were prompted by using developmental vignettes (Barrows \& Feltovich 1987, Veloski et al. 2005), to measure participants' usual or 'everyday' practice (Peabody et al. 2004). The first vignette (V1) supplied minimal information, to reflect initial interpretation at the moment when the SWS score is completed. A diagnosis at this stage would indicate the inclination to 'make do' and uncover the usually hidden assumptions which are made to fill in knowledge gaps. The second vignette (V2) provided additional clinical details reflecting the range of information required to underpin a more considered, contextual interpretation of the same assessment.

For the purpose of this study, diagnosis of withdrawal syndrome is based on two core features:

1. Physical dependence on a drug therapy administered continuously for 5 or more days, or sooner if administered at high doses (Macqueen \& Bruce 2012, Harris et al. 2016)

2. Behavioural signs of withdrawal, in response to the drug(s) stopping or reducing that are not better explained by other physical, illness or environmental causes (Macqueen \& Bruce 2012, Ista et al. 2013, Harris et al. 2016).

Provision of incomplete, equivocal information was designed to reflect the 'fuzziness of unstructured real life situations' (Benner \& Tanner 1987, p.24). V1 provided no data on either of the core features of withdrawal. V2 provided data about the likelihood of physical 
dependence, but in the absence of a baseline SWS score or trend, insufficient information to establish the cause of behavioural signs.

\section{Sample/ participants}

The study was undertaken at a specialist children's hospital in England. The study participants were registered children's nurses, who undertook withdrawal assessments regularly. Purposive sampling was employed to recruit nurses from the clinical areas where withdrawal patients were usually nursed (the Paediatric Intensive Care Unit (PICU), the High Dependency Unit (HDU) and the cardiac ward). Nurses were eligible for inclusion if they undertook withdrawal assessments regularly and considered themselves familiar with the SWS tool. Nurses were recruited by poster or by word of mouth by the researcher during clinical rounds and gave written consent to participate. Interviews took place in Autumn 2013 in quiet rooms adjacent to the clinical areas.

\section{Data collection}

Demographic data included gender and experience, in years, of applying the SWS tool in practice. No further demographic data were collected, as the relationship between factors such as years since qualification, level of expertise and level of educational attainment, on decision-making is unclear (Lauri \& Salanterä 1998, Hoffman et al. 2004, Fick et al. 2007). Consideration was given to the sequence of the interview to minimise the potential impact on typical thought processes by unintentionally problematising aspects of nursing care that may be relatively routine (Jenkins et al. 2010). The first part of the interview: interpreting SWS scores and responding with treatment choices, aimed to replicate routine clinical practice using the SWS tool and reflect the largely subconscious and automatic synthesis of information nurses undertake. V1 was presented followed by V2. After reading each vignette, participants were asked to 'think aloud' whilst responding to the pre-set questions and scripted probes e.g., 'Is this patient withdrawing?' and 'How easy or difficult is it to 
decide whether the patient is withdrawing?' A list of treatment options for the patient in V2 was then presented and nurses were reminded to 'think aloud' whilst they made a decision. Options included all drugs mentioned in V2 in addition to 'no intervention' and 'another intervention'.

The second part: noticing (defining and interpreting) individual withdrawal signs was anticipated to be more cognitively taxing, possibly causing nurses to critically reflect on their current approach to and alter subsequent, withdrawal assessments. Consequently, nurses were asked not to discuss their interview experience with colleagues until the study was completed. To encourage deeper reflection on issues raised by the vignette, nurses were asked to define four pre-selected SWS terms ('insomnia', 'irritability', 'respiratory distress', 'hypertonicity') that preliminary work had identified as being differently understood by nurses at our hospital. The nurses were also asked how easy or difficult it is to decide when a patient displayed one of these four behaviours.

\section{Ethical considerations}

Ethical approval for the study was obtained from an NHS Research Ethics Committee.

\section{Data analysis}

Interviews were conducted by JC (who had training in cognitive interviews and clinical expertise in the recognition and management of withdrawal syndrome) and were audiorecorded and transcribed by a professional transcriber. 'Informal analysis', the approach proposed by (Willis 2005, p. 156) was used to identify cognitive problems with decisionmaking. Subjective interpretation is key to informal analysis, which rather than a formal coding scheme, also relies on expert judgement to identify problems (Fonteyn et al. 1993). Analysis involved two stages; firstly the identification of the decision-making processes including cognitive errors made when noticing, interpreting and responding in individual interviews and secondly, comparison across interviews to elucidate trends. The term 
cognitive error is used to describe any flawed judgement or inaccurate decision made by the participants.

\section{Validity and reliability}

Validity and reliability were considered carefully in design and implementation of the study. A challenge in studying usual behaviour is how to do this without observation bias or research participant effects (McCambridge et al. 2014). In decision-making studies, the ideal research method has minimal impact on typical, subconscious reasoning and does not lead to an altered, more conscious level of reasoning. Research participant effects - the change in behaviour as a consequence of being studied (McCarney et al. 2007)- have been demonstrated in observational studies investigating antibiotic prescribing behaviour in paediatricians (Mangione-Smith et al. 2002) and compliance with hand hygiene in clinical settings (Eckmanns et al. 2006, Maury et al. 2006). In these studies, participants were more likely to demonstrate or take a best practice approach. In studies investigating decisionmaking, the manner of questioning may also stimulate new thinking (McCambridge et al. 2014) or change the effort paid to the cognitive task (Sitterding et al. 2012). These effects may limit the generalisability of clinical research to routine practice (McCarney et al. 2007). The cognitive interview technique is inherently suited to this study as it is not considered to alter the effort or attention paid to the task and is also widely used in psychometric testing of survey instruments (Sofaer 2002).

The vignettes were based on a real case and therefore reflected real practice and these and the verbal probes were pilot tested prior to use in the study. Their sensitivity and specificity was evident in that they generated data that identified both cognitive errors and correct decisions. The rigour of interpretive thinking and analysis was supported through dialogue and challenge by the supervisory team. 


\section{FINDINGS}

Twelve registered children's nurses participated in the interviews; four from the PICU, four from the Cardiac Ward and four from the HDU. All participants were female. The nurses had been undertaking withdrawal assessments for between 4-13 years (median 10 years) so were experienced in this aspect of their clinical role. Interviews lasted between 21-47 minutes.

In both vignettes, nurses' drew on all three options: 'withdrawing', 'not withdrawing' and 'unsure' (Table 4). In V1, two nurses recognised there was insufficient information on which to make any judgement. Responses to 'How easy or difficult was it to decide?' ranged from 'easy' to 'very difficult' with one nurse commenting that it 'should be easy with more information'. All nurses who found the diagnosis 'easy' made a definite diagnosis.

In V2, the responses to 'How easy or difficult was it to decide?' ranged from 'quite easy' to 'very difficult'. Some nurses found V2 'easier than previous [vignette]' and one thought it was 'harder with more information'. Again, those finding the diagnosis 'easy' all made a definite diagnosis. Those who found it 'easier than previous' each gave a different diagnosis. The nurse finding V2 'harder with more information' was 'unsure' in both vignettes. In terms of consistency of opinion across the vignettes, three people who made a diagnosis in V1, persisted with their diagnosis in V2 ('yes' $n=2$, 'no' $n=1$ ). Four nurses were 'unsure' in both vignettes. The two nurses who could not comment in V1 were 'unsure' in V2 and found the decision 'difficult'.

Diagnosis of withdrawal was commonly based on the SWS score in V1, although the child's underlying condition was recognised as a possible cause for the score (Table 4). In V2, more nurses recognised that the SWS score might reflect either the child's underlying conditions or their normal behaviour. Some nurses recognised that the duration of sedation described was 
too short to cause physical dependence and hence withdrawal symptoms. The three nurses who diagnosed 'not withdrawing' made this observation along with one nurse who still diagnosed the patient as 'withdrawing'. Four nurses made explicit assumptions during their deliberations in V1. Three of these nurses diagnosed withdrawal; one was 'unsure'. Two nurses made assumptions in V2. One nurse made assumptions in both vignettes and diagnosed 'withdrawal' in both cases. The second nurse was 'unsure' in both vignettes, but found V2 'easier than previous'. The common assumption in V1 was based on the length of ICU stay and related to possible sedatives the child might have received and the possibility of mechanical ventilation.

\section{Treatment response}

Treatment choices corresponded to the diagnosis when the diagnosis was definite but varied amongst nurses who were 'unsure' (Table 3 and Figure 2). Nurses who diagnosed 'withdrawing' chose to stop weaning chloral hydrate and 'maybe' increase chloral and give codeine ('yes' $n=2$, 'maybe' $n=1$ ). In contrast, nurses who diagnosed 'not withdrawing' chose to continue weaning chloral hydrate. Two nurses considered giving additional analgesia including codeine, paracetamol and oral morphine. Nurses who were 'unsure' chose a range of interventions, including stop weaning chloral hydrate, increase chloral, give codeine and no intervention. Paracetamol was chosen as 'another intervention' by five nurses who had varied opinions about whether the child was withdrawing.

Failed heuristics and biases were identified during protocol analysis and these cognitive errors were categorised according to definitions cited by Croskerry (2003) (Table 5). Cognitive errors occurred during the decision-making processes involved in both the interpretation of and response to the SWS score. Every nurse made cognitive errors: the number ranging between 1 and 4 errors per nurse. Not all cognitive errors led to diagnostic errors, as two nurses made assumptions during their deliberations in V1, but these did not 
translate into an inaccurate diagnosis. No nurse made errors at every stage of the decisionmaking process.

\section{Noticing (defining and interpreting) SWS behaviours}

Nurses shared an accurate understanding of the terms 'insomnia' and 'respiratory distress' and were confident and succinct in their definitions. They found 'irritability' harder to define, but it was usually described as difficulty in consoling the child despite trying the usual comfort measures and parental presence. 'Hypertonicity' was the most problematic term with one nurse unable to offer a definition and another giving an inaccurate definition. Although the remaining nurses offered a definition of 'increased tone', half of them expressed doubt or lacked confidence about their explanation.

When talking about the definitions, there was a tendency for nurses to blur the boundaries between signs, describing the co-existence or overlapping of some behaviours. Two nurses described the interdependence of insomnia and irritability. During a definition of 'irritability', one nurse explained 'it's linked a bit to the insomnia where you can see that they are tired and want to sleep.'(N5)inAnother nurse's definition of 'irritability' appeared to overlap with 'insomnia'; 'you sort of think they are settled, they sort of shut their eyes and they go still and then two minutes later they're awake you know, they're off again' (N10).

Inaccurate mapping of other behaviours to SWS signs was identified as another perceptual problem. Descriptions of motor disturbance were made by half of the nurses during their definitions of insomnia, irritability or hypertonicity. When defining 'insomnia' one nurse commented that 'They may be active, arms, legs, head, generally moving so they're not peacefully asleep' (N10). A definition of 'irritability' included 'thrashing their arms and legs around or their head around' (N9). 'Hypertonicity' was described as: 
Just constant moving of arms and legs, inability to stay still really, some of the babies they look like they're riding bikes lying in their cot because their legs just keep going round and their arms keep waving. (N8)

'Insomnia' presented challenges for nurses in terms of both recognising and interpreting this behaviour. Lack of familiarity with the patient made it difficult to know if the patient's behaviour was different to normal, as one nurse described 'unless you know exactly what they're like without any of the illness, medication and what have you' (N4). Trying to making sense of current behaviour by ascertaining recent trends was also complicated by the perceived subjective nature of the assessment 'if you look at the previous 12 hours, you've only got the chart to go from, so when somebody's marked down awake or asleep, you don't know if they've really been asleep for a whole hour or is it just 10 minutes' (N7). However, confidence grew throughout the shift 'because you've done a whole day with them....' (N10) and nursing a child on consecutive days was also viewed positively, because 'then you've got a better comparison as to whether they are more or less alert than they were the previous day' (N9). Environmental factors were also identified as possible causes of insomnia, as one ICU nurse described, 'ICU is noisy, it's loud, we forget and our colleagues talk and have to be shushed a lot of the time throughout the night, the monitors are always bleeping...' (N5).

The main challenge with interpreting 'irritability' related to deciding whether this behaviour was a result of withdrawal or other co-morbidities. Nurses talked about undertaking a process of eliminating other possible causes of 'irritability' before attributing it to withdrawal. As one nurse described 'it's never the first thing I think when they're crying, they might be hungry or I'll check their nappy and when I've covered all the bases then I'll be like actually they're irritable' (N12).

Lack of familiarity with the patient was raised again but some nurses described working with parents to interpret the child's behaviour, because 'they know them better than us' (N2). In 
children with neurological impairment, nurses described relying on parents to identify whether behaviours differed from normal, as one nurse explained; 'I walk into the situation and I don't know the child I might think - 'oh my word this baby's really agitated'. But the parent's might go - 'well that's him when he's well'' (N8). Nurses appeared to be most confident in recognising 'respiratory distress' but found the challenge was judging whether it was a sign of withdrawal or another co-morbidity. One nurse commented 'It's hard with the respiratory distress side of things, because if he's chronic lung disease, it's like Catch 22 isn't it?' (N6).

\section{DISCUSSION}

This paper is the first to our knowledge describing the use of cognitive interviews and vignettes to examine the stages of decision-making undertaken by nurses in the assessment and management of withdrawal syndrome. Our study showed that nurses used a variety of approaches alone or in combination including intuition, reasoning, biases and heuristics, as reported by Tanner (2006).in The use of SWS did not standardise nurses' assessment of withdrawal and cognitive challenges arose in each stage (noticing, interpreting and responding) of decision-making examined. These stages will be discussed in light of the overarching clinical goal of improving the assessment and management of withdrawal syndrome. As SWS shares a similar format and content to SOS and WAT-1, these findings suggest that cognitive challenges may also exist for nurses using SOS and WAT-1. As all nurses in the study made at least one cognitive error, there did not appear to be a relationship between quality of decision-making and either experience or their clinical specialism. Our results support the view that 'simply possessing clinical experience is no predictor of high quality decision-making' (Thompson et al. 2009, p. 610).

The noticing stage - identifying and describing individual withdrawal behaviours - presented the greatest cognitive challenge for nurses and the widest variation in responses. When asked 
to describe withdrawal signs, nurses could plainly visualise a withdrawing child, demonstrating the 'pattern recognition' of expert judgement and decision-making (Berner \& Graber 2008, p. S12). Difficulty arose in separating the component behaviours to fit a list of withdrawal signs, leading to a blurring of boundaries between terms and inaccurate mapping of other signs. Although deconstruction of withdrawal syndrome into an item pool of component behaviours may be a necessary stage in scale development (DeVellis 2012), in the experimental conditions of this study, this step appears to add complexity rather than simplifying the assessment.

Nurses recognised that they lacked knowledge needed to interpret some SWS items, as they were mostly not cognisant of the child's normal behaviour. Knowing the patient and their pattern of responses is considered fundamental to sound clinical judgement (Tanner 2006) promoting a corresponding sense of salience (Benner \& Tanner 1987), whilst less knowledge impacts on the capacity to notice subtle cues or changes. Although no reflection on the nurses in this study, this deficit in personal knowledge of the child is a limitation in the application of SWS. Accurate withdrawal assessment relies not only on a shared meaning of clinical terms but also on a shared interpretation of these behaviours in each patient. Despite the close observation possible in critical care, recognising subtle behavioural changes is more challenging in an unfamiliar patient. Including parents routinely in the assessment may benefit the process in identifying a personalised baseline of behaviours on which to consider new signs or identify trends, an approach endorsed in other complex clinical situations such as delirium (Schieveld et al. 2009) and pain assessment in children with severe neurological disability (Hunt et al. 2004).

Interpretation of the vignettes differed widely, despite every nurse being presented with the same information and clinical cues. This variation in decision-making in the face of identical information mirrors other studies involving nurses and pain assessment (Hodgins 2002), 
nurses and critical event risk assessment (Thompson et al. 2009) and triage assessments made by emergency nurses Cioffi (1998). These findings support the view that clinical judgements are influenced more by what nurses bring to the situation than by the clinical data available to them (Tanner 2006). The effort required to reach a diagnosis also varied widely: nurses who made a definite diagnosis found the decision easier than those who were unsure. For some nurses in V1, the score alone gave a clear diagnosis of withdrawal, abnegating the cognitive burden of interpreting the meaning of ambiguous clinical signs. Indeed Benner \& Tanner (1987) warned against the over-reliance on assessment tools, which could encourage a complacent 'checklist mentality' rather than the rigour of 'active enquiry'.

The ability to see some aspects as more important than others has also been described as a sense of salience by Sitterding and colleagues (2012): this sense of salience was lacking amongst nurses who overlooked the fundamental importance of recent drug history as the context for a withdrawal assessment. In the face of such complexity and the need to consciously consider the context of drug dependence, the role for the subconscious cognitive processing characteristic of intuitive thinking is unclear. Nurses who were unable to reach a diagnosis found the task harder, reflecting their recognition of the ambiguities, complexity and incompleteness of the available information, demonstrated superior decision-making (Brannon \& Carson 2003). Some nurses made probability judgements (possibly, probably or maybe withdrawing), which also inferred a cognitive flexibility to modify their opinion in light of further information (Szolovits \& Pauker 1978). Whether as a result of complacency, overconfidence or a checklist mentality, this study suggests that some nurses have a misplaced confidence in the diagnostic capacity of SWS, which would consequently limit further enquiry. The potential for cognitive errors during this interpretive phase highlights the importance of learning clinical reasoning skills, ideally during nurse training (LevettJones et al. 2010, 2015). 
The responding stage was the most consistent phase of decision-making with treatment decisions corresponding to nurses' definite diagnoses. Cioffi (1999) describes the relationship between cues and inferences as decision rules or 'if...then' rules. For example, 'If a patient is withdrawing (cues) then the drug reductions should cease (inference)' or 'If a patient is not withdrawing, then drug weaning should continue.' However, when nurses were unsure of the diagnosis, an inclination towards 'doing something' meant the most common intervention was to stop weaning chloral hydrate. This tendency towards action rather than inaction, despite no supporting evidence for the decision, is commission bias (Croskerry 2003). Unnecessary slowing of weaning regimes should be avoided however, as prolonging sedative treatment may prolong recovery and hospitalisation. Administration of analgesics was another common treatment choice made by nurses, regardless of withdrawal diagnosis, perhaps reflecting an 'obligation towards beneficence' another example of commission bias (Croskerry 2003) - despite no supporting evidence of the need for analgesia.

\section{LIMITATIONS}

This study has several limitations. Whilst the cognitive interview technique is unique in revealing cognitive processes in participants, results are not generalizable to a wider population. The interviewer works as a nurse specialist in the hospital where the study took place and was known to the nurses participating in the interviews and they identified themselves as competent in using the SWS: these factors may have affected the nurses' responses.

The bedside treatment schedule of withdrawal in the study hospital includes a guidance to stop weaning with SWS scores between 3 and 6 . The treatment schedule was not presented or discussed but it may be that some nurses recalled that a score of 5 linked to guidance to stop weaning. The number of withdrawal diagnoses in V1 may have been influenced by the fact 
that the participants were aware that the study was addressing sedation withdrawal; this might have created a diagnostic strategy of 'going for the obvious' that may not reflect typical decision-making.

\section{CONCLUSION}

This study using cognitive interviews with vignettes has provided insight into nurses' judgement and decision-making in a complex and ambiguous clinical situation. Focussing on the whole decision-making process (noticing, interpreting and responding) identified a significant cognitive burden and the potential for cognitive error at each stage. The use of a withdrawal assessment tool did not appear to simplify the process or reduce the burden.

There appears to be an inherent flaw in relying on a behavioural assessment using nonspecific signs in a population where knowledge of usual behaviour is an essential prerequisite. Including parents in the assessment may expedite recognition of behavioural changes or trends. Key areas for improvement are in recognising the clinical context necessary for withdrawal and minimising the use of biases and failed heuristics. Revealing typical thought processes provides opportunity to reflect on complex cases, which may help to support critical thinking and reduce cognitive errors. 


\section{REFERENCES}

Banning, M., 2008. A review of clinical decision making: models and current research. J. Clin. Nurs. 17, 187-195. doi:10.1111/j.1365-2702.2006.01791.x

Benner, P., Tanner, C., 1987. Clinical judgment: how expert nurses use intuition. Am. J. Nurs. 87, 23-31.

Berner, E.S., Graber, M.L., 2008a. Overconfidence as a Cause of Diagnostic Error in Medicine. Am. J. Med. 121, S2-S23. doi:10.1016/j.amjmed.2008.01.001

Best, K.M., Asaro, L.A., Franck, L.S., Wypij, D., Curley, M.A.Q., Randomized Evaluation of Sedation Titration for Respiratory Failure Baseline Study Investigators, 2016. Patterns of Sedation Weaning in Critically Ill Children Recovering From Acute Respiratory Failure. Pediatr. Crit. Care Med. J. Soc. Crit. Care Med. World Fed. Pediatr. Intensive Crit. Care Soc. 17, 19-29. doi:10.1097/PCC.0000000000000572

Brattebo, Guttorm, 2009. Training teamwork using simulation., in: Chiche, J.D., Moreno, R., Putensen, C., Rhodes, A. (Eds.), Patient Safety and Quality of Care in Intensive Care Medicine. MWV Medizinisch Wissenschaftliche Verlagsgesellschaft OHG, Berlin, pp. 93-101.

Cioffi, J., 1998. Decision making by emergency nurses in triage assessments. Accid. Emerg. Nurs. 6, 184-191.

Cook, B.G., Rumrill, P.D., 2005. Using and interpreting analogue designs. Work Read. Mass 24, 93-97.

Cranley, L., Doran, D.M., Tourangeau, A.E., Kushniruk, A., Nagle, L., 2009. Nurses’ Uncertainty in Decision-Making: A Literature Review. Worldviews Evid. Based Nurs. 6, 3-15. doi:10.1111/j.1741-6787.2008.00138.x

Croskerry, P., 2003. The importance of cognitive errors in diagnosis and strategies to minimize them. Acad. Med. J. Assoc. Am. Med. Coll. 78, 775-780. 
Cunliffe, M., McArthur, L., Dooley, F., 2004a. Managing sedation withdrawal in children who undergo prolonged PICU admission after discharge to the ward. Pediatr. Anesth. 14, 293-298. doi:10.1046/j.1460-9592.2003.01219.x

DeVellis, R.F., 2003. Scale development: theory and applications. SAGE.

DiBenedetti, D.B., Price, M.A. andrews, E.B., 2013. Cognitive interviewing in risk minimization survey development: patient and healthcare professional surveys. Expert Rev. Clin. Pharmacol. 6, 369-373. doi:10.1586/17512433.2013.811824

Easley, R.B., Nichols, D.G., 2008a. Withdrawal assessment in the pediatric intensive care unit: Quantifying a morbidity of pain and sedation management in the critically ill child*: Crit. Care Med. 36, 2479-2480. doi:10.1097/CCM.0b013e31817c0e07

Eckmanns, T., Bessert, J., Behnke, M., Gastmeier, P., Ruden, H., 2006. Compliance with antiseptic hand rub use in intensive care units: the Hawthorne effect. Infect. Control Hosp. Epidemiol. 27, 931-934. doi:10.1086/507294

Elstein, A.S., 1999. Heuristics and biases: selected errors in clinical reasoning. Acad. Med. J. Assoc. Am. Med. Coll. 74, 791-794.

Endsley, M.R., 1995. Toward a Theory of Situation Awareness in Dynamic Systems. Hum. Factors J. Hum. Factors Ergon. Soc. 37, 32-64. doi:10.1518/001872095779049543

Fick, D.M., Hodo, D.M., Lawrence, F., Inouye, S.K., 2007. Recognizing Delirium Superimposed on Dementia. J. Gerontol. Nurs. 33, 40-49.

Fonteyn, M.E., Kuipers, B., Grobe, S.J., 1993. A Description of Think Aloud Method and Protocol Analysis. Qual. Health Res. 3, 430-441. doi:10.1177/104973239300300403

Franck, L., Vilardi, J., 1995. Assessment and management of opioid withdrawal in ill neonates. Neonatal Netw. NN 14, 39-48.

Franck, L.S., Harris, S.K., Soetenga, D.J., Amling, J.K., Curley, M.A.Q., 2008. The Withdrawal Assessment Tool - Version 1 (WAT-1). Pediatr. Crit. Care Med. J. Soc. 
Crit. Care Med. World Fed. Pediatr. Intensive Crit. Care Soc. 9, 573-580. doi:10.1097/PCC.0b013e31818c8328

Gaba, D.M., Howard, S.K., Small, S.D., 1995. Situation awareness in anesthesiology. Hum. Factors 37, 20-31.

Gobet, F., Chassy, P., 2008. Towards an alternative to Benner's theory of expert intuition in nursing: a discussion paper. Int. J. Nurs. Stud. 45, 129-139. doi:10.1016/j.ijnurstu.2007.01.005

Graber, M., Gordon, R., Franklin, N., 2002. Reducing diagnostic errors in medicine: what's the goal? Acad. Med. J. Assoc. Am. Med. Coll. 77, 981-992.

Graber, M.L., Franklin, N., Gordon, R., 2005. Diagnostic error in internal medicine. Arch. Intern. Med. 165, 1493-1499. doi:10.1001/archinte.165.13.1493

Harbison, J., 2006. Clinical judgement in the interpretation of evidence: a Bayesian approach. J. Clin. Nurs. 15, 1489-1497. doi:10.1111/j.1365-2702.2005.01487.x

Harris, J., Ramelet, A.-S., van Dijk, M., Pokorna, P., Wielenga, J., Tume, L., Tibboel, D., Ista, E., 2016. Clinical recommendations for pain, sedation, withdrawal and delirium assessment in critically ill infants and children: an ESPNIC position statement for healthcare professionals. Intensive Care Med. 42, 972-986. doi:10.1007/s00134-0164344-1

Hodgins, M.J., 2002. Interpreting the meaning of pain severity scores. Pain Res. Manag. J. Can. Pain Soc. J. Société Can. Pour Trait. Douleur 7, 192-198.

Hoffman, K., Donoghue, J., Duffield, C., 2004. Decision-making in clinical nursing: investigating contributing factors. J. Adv. Nurs. 45, 53-62.

Hoffman, K.A., Aitken, L.M., Duffield, C., 2009. A comparison of novice and expert nurses' cue collection during clinical decision-making: verbal protocol analysis. Int. J. Nurs. Stud. 46, 1335-1344. doi:10.1016/j.ijnurstu.2009.04.001 
Hunt, A., Goldman, A., Seers, K., Crichton, N., Mastroyannopoulou, K., Moffat, V., Oulton, K., Brady, M., 2004. Clinical validation of the paediatric pain profile. Dev. Med. Child Neurol. 46, 9-18.

Ista, E., de Hoog, M., Tibboel, D., Duivenvoorden, H.J., van Dijk, M., 2013. Psychometric evaluation of the Sophia Observation withdrawal symptoms scale in critically ill children. Pediatr. Crit. Care Med. J. Soc. Crit. Care Med. World Fed. Pediatr. Intensive Crit. Care Soc. 14, 761-769. doi:10.1097/PCC.0b013e31829f5be1

Ista, E., van Dijk, M., de Hoog, M., Tibboel, D., Duivenvoorden, H.J., 2009. Construction of the Sophia Observation withdrawal Symptoms-scale (SOS) for critically ill children. Intensive Care Med. 35, 1075-1081. doi:10.1007/s00134-009-1487-3

Jenkins, I., 2002. The provision of analgesia and sedation in the PICU: current practice and recent advances. Pediatr. Anesth. 12, 837-839. doi:10.1046/j.14609592.2002.10271_31.x

Jenkins, N., Bloor, M., Fischer, J., Berney, L., Neale, J., 2010. Putting it in context: the use of vignettes in qualitative interviewing. Qual. Res. 10, 175-198. doi:10.1177/1468794109356737

Kahneman, D., 2011. Thinking, fast and slow. Macmillan.

Kahneman, D., Klein, G., 2009. Conditions for intuitive expertise: a failure to disagree. Am. Psychol. 64, 515-526. doi:10.1037/a0016755

Kuhlthau, CC., 1993. A principle of uncertainty for information seeking. J. Doc. 49, 339355. doi:10.1108/eb026918

Lauri, S., Salanterä, S., 1998. Decision-making models in different fields of nursing. Res. Nurs. Health 21, 443-452.

Levett-Jones, T. andersen, P., Reid-Searl, K., Guinea, S., McAllister, M., Lapkin, S., Palmer, L., Niddrie, M., 2015. Tag team simulation: An innovative approach for promoting 
active engagement of participants and observers during group simulations. Nurse Educ. Pract. 15, 345-352. doi:10.1016/j.nepr.2015.03.014

Levett-Jones, T., Hoffman, K., Dempsey, J., Jeong, S.Y.-S., Noble, D., Norton, C.A., Roche, J., Hickey, N., 2010. The 'five rights' of clinical reasoning: an educational model to enhance nursing students' ability to identify and manage clinically 'at risk' patients. Nurse Educ. Today 30, 515-520. doi:10.1016/j.nedt.2009.10.020

Lyneham, J., Parkinson, C., Denholm, C., 2008. Intuition in emergency nursing: a phenomenological study. Int. J. Nurs. Pract. 14, 101-108. doi:10.1111/j.1440172X.2008.00672.x

Macqueen, S., Bruce, E., 2012. The Great Ormond Street Hospital Manual of Children's Nursing Practices. John Wiley \& Sons.

Mangione-Smith, R., Elliott, M.N., McDonald, L., McGlynn, E.A., 2002. An Observational Study of Antibiotic Prescribing Behavior and the Hawthorne Effect. Health Serv. Res. 37, 1603-1623. doi:10.1111/1475-6773.10482

Maury, E., Moussa, N., Lakermi, C., Barbut, F., Offenstadt, G., 2006. Compliance of health care workers to hand hygiene: awareness of being observed is important. Intensive Care Med. 32, 2088-2089. doi:10.1007/s00134-006-0398-9

McCambridge, J., Witton, J., Elbourne, D.R., 2014. Systematic review of the Hawthorne effect: New concepts are needed to study research participation effects. J. Clin. Epidemiol. 67, 267-277. doi:10.1016/j.jclinepi.2013.08.015

McCarney, R., Warner, J., Iliffe, S., van Haselen, R., Griffin, M., Fisher, P., 2007. The Hawthorne Effect: a randomised, controlled trial. BMC Med. Res. Methodol. 7, 30. doi:10.1186/1471-2288-7-30 
Peabody, J.W., Luck, J., Glassman, P., Jain, S., Hansen, J., Spell, M., Lee, M., 2004. Measuring the quality of physician practice by using clinical vignettes: a prospective validation study. Ann. Intern. Med. 141, 771-780.

Rew, L., Barrow, E.M., 2007. State of the science: intuition in nursing, a generation of studying the phenomenon. ANS Adv. Nurs. Sci. 30, E15-25.

Ross, L., Lundstrøm, L.H., Petersen, M.A., Johnsen, A.T., Watt, T., Groenvold, M., 2012. Using method triangulation to validate a new instrument (CPWQ-com) assessing cancer patients' satisfaction with communication. Cancer Epidemiol. 36, 29-35. doi:10.1016/j.canep.2011.09.002

Schieveld, J.N.M., van der Valk, J.A., Smeets, I., Berghmans, E., Wassenberg, R., Leroy, P.L.M.N., Vos, G.D., van Os, J., 2009. Diagnostic considerations regarding pediatric delirium: a review and a proposal for an algorithm for pediatric intensive care units. Intensive Care Med. 35, 1843-1849. doi:10.1007/s00134-009-1652-8

Simmons, B., Lanuza, D., Fonteyn, M., Hicks, F., Holm, K., 2003. Clinical reasoning in experienced nurses. West. J. Nurs. Res. 25, 701-719-724.

Sitterding, M.C., Broome, M.E., Everett, L.Q., Ebright, P., 2012. Understanding situation awareness in nursing work: a hybrid concept analysis. ANS Adv. Nurs. Sci. 35, 7792. doi:10.1097/ANS.0b013e3182450158

Sofaer, S., 2002. Qualitative research methods. Int. J. Qual. Health Care 14, 329-336. doi:10.1093/intqhe/14.4.329

Szolovits, P., Pauker, G., 1978. Categorical and probabilistic reasoning in medical diagnosis. Artif. Intell. 11, 115-144.

Tanner, C.A., 2006. Thinking like a nurse: a research-based model of clinical judgment in nursing. J. Nurs. Educ. 45, 204-211. 
Thompson, C., Bucknall, T., Estabrookes, C.A., Hutchinson, A., Fraser, K., de Vos, R., Binnecade, J., Barrat, G., Saunders, J., 2009. Nurses' critical event risk assessments: a judgement analysis. J. Clin. Nurs. 18, 601-612. doi:10.1111/j.13652702.2007.02191.x

Tourangeau, R., Rips, L.J., Rasinski, K., 2000. The Psychology of Survey Response. Cambridge University Press.

Twycross, A., Powls, L., 2006. How do children's nurses make clinical decisions? Two preliminary studies. J. Clin. Nurs. 15, 1324-1335. doi:10.1111/j.13652702.2006.01453.x

Veloski, J., Tai, S., Evans, A.S., Nash, D.B., 2005. Clinical vignette-based surveys: a tool for assessing physician practice variation. Am. J. Med. Qual. Off. J. Am. Coll. Med. Qual. 20, 151-157. doi:10.1177/1062860605274520

Willis, G.B., 2005. Cognitive Interviewing. A Tool for Improving Questionnaire Design. Sage Publications Inc. 